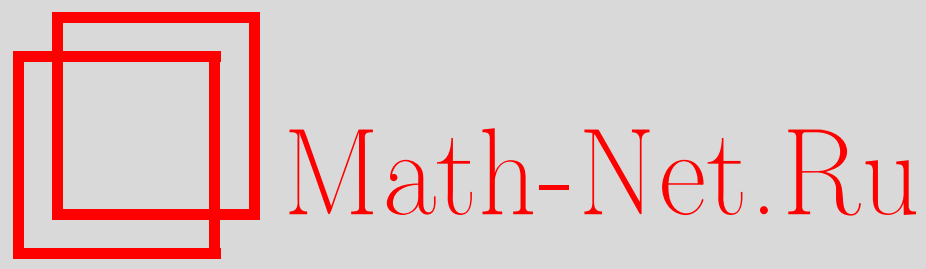

А. Г. Костюченко, К. А. Мирзоев, Обобщенные якобиевы матрицы и индексы дефекта обыкновенных дифференциальных операторов с полиномиальными коэффициентами, Функи. анализ и его прил., 1999, том 33, выпуск 1, 30-45

DOI: https://doi.org/10.4213/faa336

Использование Общероссийского математического портала MathNet.Ru подразумевает, что вы прочитали и согласны с пользовательским соглашением

http://www . mathnet.ru/rus/agreement

Параметры загрузки:

IP : 3.93 .64 .190

26 апреля 2023 г., 03:25:31

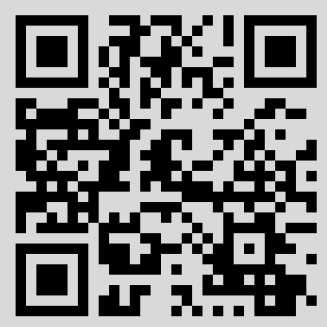




\title{
Обобщенные якобиевы матрицы и индексы дефекта обыкновенных дифференциальных операторов с полиномиальными коэффициентами *
}

\author{
(C) 1999. А. Г. Костюченко, К. А. МиРзоев
}

Светлой памяти Мищи Юркина посвящаем

В настоящей работе исследуются задачи об индексах дефекта якобиевых матриц с квадратными матричными элементами и обыкновенных симметрических дифференциальных операторов с полиномиальными коэффициентами в пространствах $l_{2}$ и $\mathscr{L}_{2}(-\infty,+\infty)$ соответственно. Как известно, якобиевы матрицы с матричными элементами возникают, например, в связи с матричной проблемой моментов. В данной статье, в частности, найден критерий того, что якобиева матрица с матричными элементами специального вида является вполне неопределенной (определение см. ниже). Из него следует, что операторы, порожденные вполне неопределенными якобиевыми матрицами в пространстве $l_{2}$, являются целыми операторами минимального типа (см. $[1,2])$. Следовательно, самосопряженные расширения этих операторов имеют дискретный спектр, спектральные меры — определенную структуру и т.д.

Еще одним источником возникновения подобных якобиевых матриц является матричное представление обыкновенных симметрических дифференциальных операторов с полиномиальными коэффициентами на всей оси. Таким образом обнаруживаются новые классы целых операторов минимального типа с определенными дефектными числами. При этом дефектные числа таких операторов могут быть меньше или равны, но - что особенно интересно - могут оказаться и больше порядка соответствующего дифференциального выражения.

Таким образом, оказывается, что открытый М. Г. Крейном в $[1,2]$ класс целых операторов значительно шире, чем ранее предполагалось.

\section{§0. Предварительные сведения}

1. Пусть $\mathbb{C}^{p}(p \geqslant 1)$ - евклидово $p$-мерное пространство вектор-столбцов со стандартным скалярным произведением $y^{*} x=\sum_{j=1}^{p} x_{j} \bar{y}_{j}$, где комплексные числа $x_{j}, y_{j}(j=1, \ldots, p)$ - координаты векторов $x$ и $y$ соответственно, а звездочка означает комплексно-сопряженную матрицу. Пусть далее $A_{j}, B_{j}(j=0,1, \ldots)$ - квадратные матрицы порядка $p$, элементы кото-

* Работа выполнена при поддержке РФФИ, грант № 96-01-00333 и грант поддержки научных школ № 96-15-01292. 
рых являются комплексными числами, причем $A_{j}$ - самосопряженные матрицы и $B_{j}^{-1}(j=0,1, \ldots)$ существуют. Бесконечную матрицу

$$
\mathbf{J}=\left(\begin{array}{ccccc}
A_{0} & B_{0} & O & O & \ldots \\
B_{0}^{*} & A_{1} & B_{1} & O & \ldots \\
O & B_{1}^{*} & A_{2} & B_{2} & \ldots \\
\vdots & \vdots & \vdots & \vdots & \ddots
\end{array}\right),
$$

где $O$ - нулевая матрица порядка $p$, назовем якобиевой матрицей с матричными элементами. Обозначим через $l_{p}^{2}$ гильбертово пространство бесконечных последовательностей $u=\left(u_{0}, u_{1}, \ldots\right), u_{j} \in \mathbb{C}^{p}$, со скалярным произведением $(u, v)=\sum_{j=0}^{+\infty} v_{j}^{*} u_{j}$. Матрица $\mathbf{J}$ посредством операций

$$
(l u)_{j}=B_{j} u_{j+1}+A_{j} u_{j}+B_{j-1}^{*} u_{j-1}, \quad j=0,1, \ldots,
$$

где $u_{-1}=0, u_{0}, u_{1}, \cdots \in \mathbb{C}^{p}$, определяет на многообразии всех финитных векторов (т.е. векторов с конечным числом ненулевых векторов-координат) пространства $l_{p}^{2}$ симметрический оператор, замыкание которого мы обозначим через $L$. Хорошо известно, что $L$ - минимальный замкнутый симметрический оператор, порожденный выражением (0.1) и граничным условием $u_{-1}=0$ в пространстве $l_{p}^{2}$, — вообще говоря, несамосопряжен и дефектные числа $n_{+}$и $n_{-}$этого оператора удовлетворяют неравенствам $0 \leqslant n_{+}$, $n_{-} \leqslant p($ см. $[1-3$, гл. VII, §2]).

Согласно М. Г. Крейну [1, 2], матричная проблема моментов, порождаемая матрицей $\mathbf{J}$, называется вполне неопределенной, если $n_{+}=n_{-}=p$. Несколько упрощая терминологию, в этом случае мы будем говорить, что для матрицы $\mathbf{J}$ (для выражения $l$, для оператора $L$ ) имеет место вполне неопределенный случай. В [4], в частности, установлено, что справедлива

ТЕОРемА 0.1. Для матрицьи Ј тогда и только тогда имеет место вполне неопределенный случай, когда все решения векторного уравнения

$$
(l u)_{j}=z u_{j}, \quad j=1,2, \ldots,
$$

при $z=0$ принадлежат пространству $l_{p}^{2}$.

В 11 данной работы, исходя из теоремы 0.1, получен эффективный критерий того, что обобщенная якобиева матрица простейшего вида является вполне неопределенной (см. п. 1, 1 ).

2. Бесконечную матрицу $\mathbf{J}_{n}$ с квадратными матричными элементами $C_{i j}$ $(i, j=0,1, \ldots)$ порядка $p$ назовем обобщенной якобиевой матрицей порядка $2 n$, если $C_{i j}=C_{j i}^{*}, C_{i j}=O$ при $|i-j|>n$ и $C_{j, j+n} \neq O$. Обобщенная якобиева матрица $\mathbf{J}_{n}$, очевидно, имеет ту же структуру, что и $\mathbf{J}$ с матричными элементами $A_{j}$ и $B_{j}$ размера $n p \times n p$, но некоторые из матриц $B_{j}$ могут оказаться необратимыми. Однако и в этом случае с матрицей $\mathbf{J}_{n}$ естественным образом можно связать операцию $l_{n}$, аналогичную операции $(0.1)$. В терминах элементов $C_{i j}$ операция $l_{n}$ имеет вид

$$
\left(l_{n} u\right)_{j}=\sum_{k=-n}^{n} C_{j, j+k} u_{j+k}, \quad j=0,1, \ldots,
$$


где $u_{-n}=u_{-n+1}=\cdots=u_{-1}=0, u_{0}, u_{1}, \ldots \in \mathbb{C}^{p}$. Обозначим через $L_{n}$ минимальный замкнутый симметрический оператор, порожденный выражением $(0.3)$ в пространстве $l_{p}^{2}$. Нетрудно видеть, что дефектные числа $n_{+}$и $n_{-}$оператора $L_{n}$ удовлетворяют неравенствам $0 \leqslant n_{+}, n_{-} \leqslant n p$. Действительно, существует, очевидно, вешественное число $\mu$, такое, что матрицы $\widetilde{C}_{j, j+n}=C_{j, j+n}+\mu I(j=0,1, \ldots)$, где $I$ - единичная матрица, обратимы. Заметив это, обозначим через $\widetilde{\mathbf{J}}_{n}$ якобиеву матрицу, полученную из $\mathbf{J}_{n}$ заменой элементов $C_{j, j+n}$ (вслед за ними и $\left.C_{j, j-n}\right)$ на $\widetilde{C}_{j, j+n}$. Дефектные числа $\tilde{n}_{+}$и $\tilde{n}_{-}$оператора $\widetilde{L}_{n}$, соответствующего матрице $\widetilde{\mathbf{J}}_{n}$, удовлетворяют неравенствам $0 \leqslant \tilde{n}_{+}, \tilde{n}_{-} \leqslant n p$. С другой стороны, разность $L_{n}-\widetilde{L}_{n}$ является ограниченным самосопряженным оператором в $l_{p}^{2}$. Поэтому, согласно известной теореме (см., например, [5, т. 2, гл. VIII, п. 100, предложение $\left.4^{\circ}\right]$ ), $n_{+}=\tilde{n}_{+}$и $n_{-}=\tilde{n}_{-}$.

Таким образом, определения и факты п. 1 применимы и для обобщенных якобиевых матриц $\mathbf{J}_{n}$. В $\S \S 1,2$ настоящей работы исследуются дефектные числа оператора $L_{n}$ в терминах элементов $C_{i j}$ матрицы $\mathbf{J}_{n}$.

3. Пусть $l$ - формально самосопряженное дифференциальное выражение четного или нечетного порядка $r$ на прямой вида

$$
l=\sum_{j=0}^{r} q_{j}(x) \frac{d^{j}}{d x^{j}}
$$

где $q_{j}(j=0,1, \ldots, r)$ - заданные полиномы от переменного $x$. Оператор, порождаемый выражением $l$ на финитных бесконечно дифференцируемых функциях на $(-\infty,+\infty)$, является симметрическим в пространстве квадратично интегрируемых функций $\mathscr{L}_{2}\left(\equiv \mathscr{L}_{2}(-\infty,+\infty)\right)$ и поэтому допускает замыкание. Символом $L$ обозначим это замыкание. Матричное представление оператора $L$ в некотором базисе имеет вид якобиевой матрицы Ј (см. п. 1 $\S 3$ настоящей работы). Поэтому мы сохранили одинаковые обозначения для выражений (0.1) и (0.4) и соответствующих им минимальных операторов.

Рассмотрим дифференциальные выражения

$$
a=\frac{1}{\sqrt{2}}\left(x+\frac{d}{d x}\right), \quad a^{*}=\frac{1}{\sqrt{2}}\left(x-\frac{d}{d x}\right) .
$$

На множестве финитных бесконечно дифференцируемых функций выполняются соотношения

$$
x=\frac{1}{\sqrt{2}}\left(a+a^{*}\right), \quad \frac{d}{d x}=\frac{1}{\sqrt{2}}\left(a-a^{*}\right), \quad a a^{*}-a^{*} a=E,
$$

где $E$ - единичный оператор в $\mathscr{L}_{2}$. Из этих соотношений следует, что выражение (0.4) можно представить в виде

$$
l=\sum_{s+t \leqslant n} h_{s t} a^{*^{s}} a^{t}, \quad h_{s t}=\bar{h}_{t s},
$$

где $h_{s t} \in \mathbb{C}$ и $n$ - натуральное число. Отметим, что число $n$, вообще говоря, отлично от $r$. Кроме того, от чисел $h_{s t}$ требуется лишь, чтобы $h_{s, s-n} \neq 0$ 
при некотором $s \in\{0,1, \ldots, n\}$. Эти обстоятельства, как правило, затрудняют работу с представлением (0.5) для выражения $l$. C другой стороны, именно представление (0.5) в некоторых случаях позволяет найти дефектные числа оператора $L$. Небольшой $\S 3$ настоящей работы посвящен дефектным числам оператора $L$ в терминах чисел $n$ и $h_{s t}$.

Работы [6] и [7] также посвящены индексам дефекта операторов, порожденных обобщенными якобиевыми матрицами. В частности, в работе [7] асимптотическими методами для конечно-разностных уравнений исследуются дефектные числа операторов, порожденных выражениями вида $(0.3)$ и $(0.5)$.

\section{§1. Об обобщениях теоремы Березанского о неопределенности якобиевых матриц}

1. Предположим, что обобщенная симметрическая якобиева матрица $\mathbf{J}_{m}^{(0)}$ имеет только две ненулевые диагонали, а именно, пусть элементы $C_{i j}$ этой матрицы удовлетворяют следующим условиям: $C_{i j}=O$, если $|i-j| \neq m$, $C_{i, i+m}=B_{i}$ и $C_{i j}=C_{j i}^{*}(i, j=0,1, \ldots)$, где $m$ - фиксированное натуральное число, а $B_{i}$ - последовательность обратимых матриц порядка $p$. Символом $\|\cdot\|$ обозначим спектральную матричную норму. Справедлива следующая теорема:

ТЕорема 1.1. Для якобиевой матрицьл $\mathbf{J}_{m}^{(0)}$ тогда и только тогда имеет место вполне неопределенный случай, когда ее матричные элементь $B_{i}$ nри $s=0,1, \ldots, 2 m-1$ удовлетворяют условиям

$$
\sum_{j=1}^{+\infty}\left\|B_{(2 j-1) m+s}^{-1} B_{(2 j-2) m+s}^{*} \cdots B_{m+s}^{-1} B_{s}^{*}\right\|^{2}<+\infty .
$$

ДокАЗАтЕЛьство. Матричное конечно-разностное уравнение, соответствующее векторному уравнению (0.2) (при $z=0)$, имеет вид

$$
B_{j} U_{j+m}+B_{j-m}^{*} U_{j-m}=O, \quad j=m, m+1, \ldots .
$$

Обозначим через $P_{j}^{(s)}(s=0,1, \ldots, 2 m-1, j=0,1, \ldots)$ решения уравнения (1.2), удовлетворяющие начальным условиям

$$
U_{0}=U_{1}=\cdots=U_{s-1}=U_{s+1}=\cdots=U_{2 m-1}=O, \quad U_{s}=C_{s},
$$

где

$$
C_{s}= \begin{cases}B_{s}^{*-1}, & s=0,1, \ldots, m-1, \\ I, & s=m, m+1, \ldots, 2 m-1 .\end{cases}
$$

Последовательности $P_{j}^{(s)}$ вычисляются явно из уравнения $(1.2)$ :

$$
P_{2 j m+k}^{(s)}= \begin{cases}O, & k \neq s, \\ (-1)^{j} B_{(2 j-1) m+s}^{-1} B_{(2 j-2) m+s}^{*} \cdots B_{m+s}^{-1} B_{s}^{*} C_{s}, & k=s .\end{cases}
$$


Из равенств (1.4) следует, что

$$
\sum_{j=0}^{+\infty}\left\|P_{j}^{(s)}\right\|^{2}<+\infty, \quad s=0,1, \ldots, 2 m-1
$$

тогда и только тогда, когда выполняются условия (1.1) теоремы 1.1. Это, в свою очередь, очевидно, равносильно утверждению о том, что все решения уравнения (0.2) принадлежат пространству $l_{p}^{2}$. Таким образом, применяя теорему 0.1 , завершаем доказательство теоремы 1.1.

2. В нашей работе [4] приведены признаки того, что якобиева матрица J является не вполне неопределенной, в терминах элементов $A_{j}$ и $B_{j}$ этой матрицы. Здесь же приведем несколько следствий теоремы 1.1 , в которых утверждается, что для матрицы $\mathbf{J}_{m}^{(0)}$ при определенных условиях на ее элементы $B_{j}$ имеет место вполне неопределенный случай. Применяя признак Даламбера к рядам в левой части условий (1.1), приходим к выводу, что справедливо

СЛЕДСТВИЕ 1.1. Пусть

$$
\lim _{j \rightarrow \infty} \frac{\left\|B_{(2 j+1) m+s}^{-1} B_{2 j m+s}^{*} \cdots B_{m+s}^{-1} B_{s}^{*}\right\|}{\left\|B_{(2 j-1) m+s}^{-1} B_{(2 j-2) m+s}^{*} \cdots B_{m+s}^{-1} B_{s}^{*}\right\|}=q_{s}, \quad s=0,1, \ldots, 2 m-1 .
$$

Если $q_{s}<1$ для любого $s \in\{0,1, \ldots, 2 m-1\}$, то для матриць $\mathbf{J}_{m}^{(0)}$ имеет место вполне неопределенный случай; если же $q_{s}>1$ при некотором $s \in$ $\{0,1, \ldots, 2 m-1\}$, то для матриць $\mathbf{J}_{m}^{(0)}$ вполне неопределенньй случай места не имеет.

Применяя теперь кольцевое свойство матричной нормы, получаем, что общие члены рядов в левой части условий (1.1) не превосходят соответственно величин

$$
\left\|B_{s} B_{m+s}^{*-1}\right\|^{2} \cdots\left\|B_{(2 j-2) m+s} B_{(2 j-1) m+s}^{*-1}\right\|^{2} .
$$

Используя далее признаки сходимости рядов с общими членами (1.5), можно найти различные достаточные условия выполнимости условий (1.1). В частности, применяя признак Раабе, получаем

СЛЕдСТВИЕ 1.2. Пусть, начиная с некоторого номера $j$,

$$
\left\|B_{j} B_{j+m}^{*-1}\right\| \leqslant 1-q / j, \quad \text { 2дe } q>m .
$$

Тогда для матрицы $\mathbf{J}_{m}^{(0)}$ имеет место вполне неопределенньй случай.

Теперь докажем, что справедливо

СЛЕдСТВИЕ 1.3. Пусть, начиная с некоторого номера $j \geqslant m$,

$$
\left\|B_{j-m}\right\| \cdot\left\|B_{j+m}\right\| \leqslant\left\|B_{j}^{-1}\right\|^{-2}
$$

$u$

$$
\sum_{j=1}^{+\infty} \frac{1}{\left\|B_{j}\right\|}<+\infty
$$

Тогда для матрицы $\mathbf{J}_{m}^{(0)}$ имеет место вполне неопределенньй случай. 
ДокАЗАТЕЛЬСтво. Из условий (1.6) вытекает, что

$$
\begin{aligned}
& \left\|B_{m+s}^{-1}\right\|^{2}\left\|B_{3 m+s}^{-1}\right\|^{2} \cdots \\
& \quad \leqslant B_{(2 j-1) m+s}^{-1} \|^{2} \\
& \quad \leqslant\left\|B_{s}\right\|^{-1}\left(\left\|B_{2 m+s}\right\| \cdots\left\|B_{(2 j-2) m+s}\right\|\right)^{-2}\left\|B_{2 j m+s}\right\|^{-1} .
\end{aligned}
$$

Поэтому величины (1.5) и, следовательно, общие члены рядов из левой части условий (1.1) не превосходят $\left\|B_{s}\right\| /\left\|B_{2 j m+s}\right\|$. Остается заметить, что условие (1.7) обеспечивает сходимость рядов

$$
\left\|B_{s}\right\| \sum_{j=1}^{+\infty} \frac{1}{\left\|B_{2 j m+s}\right\|}, \quad s=0,1, \ldots, 2 m-1 .
$$

Следствие 1.3 доказано.

Отметим, что если имеют место неравенства (1.6), то условия (1.7) и

$$
\sum_{j=1}^{+\infty}\left\|B_{j}^{-1}\right\|<+\infty
$$

равносильны. Действительно, из (1.6) следует, что

$$
\left\|B_{j}^{-1}\right\| \leqslant \frac{1}{\sqrt{\left\|B_{j-m}\right\|\left\|B_{j+m}\right\|}} \leqslant \frac{1}{2}\left(\frac{1}{\left\|B_{j-m}\right\|}+\frac{1}{\left\|B_{j+m}\right\|}\right) .
$$

Поэтому из (1.7) вытекает (1.8). Обратное же утверждение справедливо всегда, поскольку $\left\|B_{j}\right\|^{-1} \leqslant\left\|B_{j}^{-1}\right\|$. Применяя далее признак Карлемана (см. $[3$, гл. VII, §2, п. 11]) и следствие 1.3, легко убедиться, что если выполнены условия (1.6), то минимальный оператор $L_{m}^{(0)}$, порожденный матрицей $\mathbf{J}_{m}^{(0)}$ (см. 0, п. 2), имеет либо максимальные, либо минимальные дефектные числа, в зависимости от того, выполняется или нет условие (1.7) (или (1.8)). Здесь промежуточные значения дефектных чисел невозможны.

\section{3. Рассмотрим два примера.}

Пусть $m=1$ и $p=1$ и элементы $b_{j}$ скалярной якобиевой матрицы $\mathbf{J}_{1}^{(0)}$ - положительные числа. Хорошо известно, что матрица $\mathbf{J}_{1}^{(0)}$ порождается классической проблемой моментов Гамбургера.

ПРИмеР 1. Пусть $a_{k}$ - произвольная числовая последовательность с положительными членами и $b_{2 k}=b_{2 k+1}=a_{k}(k=0,1, \ldots)$. Из теоремы 1.1 , очевидно, следует, что для матрицы $\mathbf{J}_{1}^{(0)}$ имеет место определенный случай, т.е. соответствующая проблема моментов определенная.

ПРИмЕР 2. Пусть $b_{2 k}=c_{1} k^{\alpha}\left(1+\alpha_{k}\right)$ и $b_{2 k+1}=c_{2} k^{\beta}\left(1+\beta_{k}\right)$, где $\alpha, \beta, c_{1}$ и $c_{2}$ - положительные постоянные и $\lim _{k \rightarrow \infty} \alpha_{k}=\lim _{k \rightarrow \infty} \beta_{k}=0$. Из следствия 1.1 вытекает, что если $\alpha \neq \beta$ или $\alpha=\beta$, но $c_{1} \neq c_{2}$, то соответствующая проблема моментов определенная. Пусть теперь $\alpha=\beta$ и $c_{1}=c_{2}$. Тогда следует учесть асимптотическое поведение последовательностей $\alpha_{k}$ и $\beta_{k}$ при $k \rightarrow \infty$. Действительно, например, в случае, когда $\alpha_{k}=a k^{-1}+O\left(k^{-1-\sigma}\right)$ и 
$\beta_{k}=b k^{-1}+O\left(k^{-1-\sigma}\right)(\sigma>0)$ при $k \rightarrow \infty$, применяя признак Гаусса сходимости рядов, из теоремы 1.1 мы получаем, что соответствующая проблема моментов является неопределенной, если $b-a>1 / 2$ и $\alpha>1 / 2+b-a$, и определенной во всех остальных случаях.

Отметим, что известные нам признаки определенности или неопределенности, полученные ранее другими авторами, не позволяют определить тип матрицы $J_{1}^{(0)}$ в случаях примеров 1 и 2. Кроме того, эти примеры хорошо иллюстрируют рамки применимости признака Карлемана и следствий 1.1-1.3.

4. Пусть теперь обобщенная якобиева матрица $\mathbf{J}_{n}(n \geqslant m)$ имеет вид $\mathbf{J}_{n}=\mathbf{J}_{m}^{(0)}+\mathbf{J}_{n}^{(1)}$, где $\mathbf{J}_{m}^{(0)}$ - матрица той же структуры, что и раньше, а элементы $C_{i j}^{(1)}$ матрицы $\mathbf{J}_{n}^{(1)}$ ограничены в совокупности, т.е. $\left\|C_{i j}^{(1)}\right\| \leqslant M$ при некотором $M>0$. Тогда оператор $L_{n}$ имеет вид $L_{n}=L_{m}^{(0)}+L_{n}^{(1)}$, где $L_{n}^{(1)}$, очевидно, - ограниченный самосопряженный оператор. Поэтому, согласно известной теореме (см., например [5, т. 2, гл. VIII, п. 10, предложение $\left.4^{\circ}\right]$ ), дефектные числа операторов $L_{n}$ и $L_{m}^{(0)}$ совпадают. Таким образом, теорема 1.1 и следствия 1.1-1.3 позволяют определить дефектные числа оператора $L_{n}$. В частности, при $n=m$, положив $m=p=1$ в следствии 1.3 , получим еще одно доказательство теоремы Ю. М. Березанского о неопределенности якобиевых матриц (см. [3, гл. VIII, §1, п. 2, теорема 1.5]).

Утверждение типа следствия 1.2 содержится в работе [6, II] (см. следствие 2.3 и п. $5 \S 2$ настоящей работы).

\section{§ 2. Индексы дефекта операторов, порожденных обобщенными якобиевыми матрицами}

1. Пусть $F_{j}(j=0,1, \ldots)$ - произвольная последовательность матриц размера $p \times p$. Методом вариации постоянных решим матричное уравнение

$$
B_{j} U_{j+m}+B_{j-m}^{*} U_{j-m}=F_{j-m}, \quad j=m, m+1, \ldots
$$

Заметим, что последовательность

$$
U_{j}=\sum_{s=0}^{2 m-1} P_{j}^{(s)} C_{j}^{(s)}
$$

удовлетворяет уравнению (2.1), если последовательности матриц $C_{j}^{(s)}(s=$ $0,1, \ldots, 2 m-1)$ удовлетворяет равенствам

$$
\begin{gathered}
B_{j}\left(\sum_{s=0}^{2 m-1} P_{j+m}^{(s)} \Delta C_{j+m-k}^{(s)}\right)=\delta_{k, 2 m-1} F_{j-m} \\
k=0,1, \ldots, 2 m-1, j=m, m+1, \ldots
\end{gathered}
$$

где $\Delta C_{j}^{(s)}=C_{j}^{(s)}-C_{j-1}^{(s)}$, а $\delta_{i j}$ - символ Кронекера.

Положив $j+m-k=l$ в равенствах (2.2), получаем, что неизвестные $\Delta C_{l}^{(s)}$ $(s=0,1, \ldots, 2 m-1)$ при фиксированном $l$ являются решениями системы 
уравнений

$$
\begin{gathered}
B_{l+k-m}\left(\sum_{s=0}^{2 m-1} P_{l+k}^{(s)} \Delta C_{l}^{(s)}\right)=\delta_{k, 2 m-1} F_{l+k-2 m}, \\
l+k \geqslant 2 m, k=0,1, \ldots, 2 m-1 .
\end{gathered}
$$

Система уравнений (2.3) при $l=2 m, 2 m+1, \ldots$ имеет единственное решение. Действительно, из формул (1.4) следует, что, во-первых, все неизвестные $\Delta C_{l}^{(s)}$ этой системы, кроме той, которая определяется из последнего уравнения $(k=2 m-1)$, равны нулевой матрице. Во-вторых, положив $l+2 m-1=2 n m+i(n=1,2, \ldots, i=0,1, \ldots, 2 m-1)$ в последнем уравнении системы (2.3), получаем, что

$$
B_{(2 n-1) m+i} P_{2 n m+i}^{(i)} \Delta C_{(2 n-2) m+i+1}^{(i)}=F_{(2 n-2) m+i} .
$$

Используя далее соотношения (1.3) и (1.4), из этого равенства легко извлечь, что

$$
\Delta C_{(2 n-2) m+i+1}^{(i)}=\left\{\begin{aligned}
-\left(P_{(2 n-1) m+i}^{(i+m)}\right)^{*} F_{(2 n-2) m+i}, & i=0,1, \ldots, m-1, \\
\left(P_{(2 n-1) m+i}^{(i-m)}\right)^{*} F_{(2 n-2) m+i}, & i=m, m+1, \ldots, 2 m-1 .
\end{aligned}\right.
$$

Таким образом, при $l=2 m, 2 m+1, \ldots$ система $(2.3)$ имеет единственное решение и справедлива формула

$$
\Delta C_{l}^{(s)}=\left\{\begin{aligned}
-\left(P_{l+m-1}^{(s+m)}\right)^{*} F_{l-1}, & s=0,1, \ldots, m-1, \\
\left(P_{l+m-1}^{(s-m)}\right)^{*} F_{l-1}, & s=m, m+1, \ldots, 2 m-1 .
\end{aligned}\right.
$$

При $l=1,2, \ldots, 2 m-1$ количество уравнений в системе (2.3) меньше, чем количество неизвестных. Однако легко показать, что и в этом случае формулы (2.4) определяют решения этой системы. Полагая теперь $l=i, i+1, \ldots, j$ в равенствах (2.4) и суммируя полученные равенства, имеем

$$
C_{j}^{(s)}=C_{i-1}^{(s)}+ \begin{cases}-\sum_{k=i+m-1}^{j+m-1}\left(P_{l}^{(s+m)}\right)^{*} F_{l-m}, & s=0,1, \ldots, m-1, \\ \sum_{k=i+m-1}^{j+m-1}\left(P_{l}^{(s-m)}\right)^{*} F_{l-m}, & s=m, m+1, \ldots, 2 m-1 .\end{cases}
$$

Таким образом, последовательность $U_{j}$ представляется в следующем виде:

$$
U_{j}=\sum_{s=0}^{2 m-1} P_{j}^{(s)} C_{i-1}^{(s)}+\sum_{l=i+m-1}^{j+m-1} K_{j l} F_{l-m}, \quad j=i, i+1, \ldots,
$$

где $C_{i-1}^{(s)}(s=1,2, \ldots, 2 m-1)$ - постоянные матрицы и

$$
K_{j l}=\sum_{s=0}^{m-1}\left[P_{j}^{(s+m)}\left(P_{l}^{(s)}\right)^{*}-P_{j}^{(s)}\left(P_{l}^{(s+m)}\right)^{*}\right]
$$


Последовательность $K_{j l}$ - последовательность Коши для уравнения (2.1), - очевидно, удовлетворяет уравнению (1.2) при фиксированном $l$ и соотношению $K_{j l}^{*}=-K_{l j}$. Кроме того, если взять $F_{j}=I(j=0,1, \ldots)$ и подставить полученные выражения для $\Delta C_{l}^{(s)}$ из формул (2.4) в систему $(2.3)$, то после некоторых элементарных преобразований, которые мы здесь не приводим, получим

$$
K_{l+k, l}=\delta_{k, m} B_{l}^{-1}, \quad k=0,1, \ldots, 2 m-1, l=0,1, \ldots .
$$

Изложенные выше рассуждения позволяют установить, что для последовательности $K_{j l}(j, l=0,1, \ldots)$ справедливы формулы, аналогичные формулам (1.4): а именно, при $p=0,1, \ldots$ имеет место равенство

$$
K_{j l}= \begin{cases}O, & j \neq l+(2 p+1) m, \\ (-1)^{p} B_{l+2 p m}^{-1} B_{l+(2 p-1) m}^{*} \cdots B_{l+m}^{*} B_{l}^{-1}, & j=l+(2 p+1) m .\end{cases}
$$

2. Пусть $\mathbf{J}_{n}^{\prime}$ - обобщенная якобиева матрица, полученная из матрицы $\mathbf{J}_{n}$ путем замены в ней первых $i$ строк и стольких же столбцов нулевыми матрицами порядка $p$, где $i$ - некоторое натуральное число. Матричное уравнение, соответствующее векторному уравнению (0.2) для матрицы $\mathbf{J}_{n}^{\prime}$, имеет вид

$$
\sum_{k=-n}^{n} C_{j, j+k} U_{j+k}=z U_{j}, \quad j=i, i+1, \ldots,
$$

где $z \in \mathbb{C}$ и $U_{i-n}=U_{i-n+1}=\cdots=U_{i-1}=O$. Уравнение $(2.8)$ преобразуем к виду

где

$$
B_{j} U_{j+m}+B_{j-m}^{*} U_{j-m}=\sum_{k=-n}^{n} C_{j k}^{\prime} U_{k}
$$

$$
\begin{gathered}
C_{j, j+k}^{\prime}=-C_{j, j+k} \quad \text { при } k \in\{-n,-n+1, \ldots, n\} \backslash\{-m, 0, m\}, \\
C_{j, j-m}^{\prime}=B_{j-m}^{*}-C_{j, j-m}, \quad C_{j j}^{\prime}=z I-C_{j j}, \quad C_{j, j+m}^{\prime}=B_{j}^{*}-C_{j, j+m} .
\end{gathered}
$$

Пусть теперь $U_{i}, U_{i+1}, \ldots, U_{i+m-1}$ - произвольные матрицы порядка $p$. В равенстве (2.5) положим

$$
F_{l-m}=\sum_{k=-n}^{n} C_{l, l+k}^{\prime} U_{l+k}
$$

и постоянные $C_{i-1}^{(s)}$ выберем так, чтобы выполнялись условия

$$
\sum_{s=0}^{2 m-1} P_{j}^{(s)} C_{i-1}^{(s)}= \begin{cases}O, & j=i-m, i-m+1, \ldots, i-1 \\ U_{j}, & j=i, i+1, \ldots, i+m-1\end{cases}
$$

Этими условиями постоянные $C_{i-1}^{(s)}(s=0,1, \ldots, 2 m-1)$, очевидно, определяются однозначно. Таким образом, матричные решения $U_{j}$ уравнения $(2.8)$ 
удовлетворяют тождеству

$U_{j}=\sum_{s=0}^{2 m-1} P_{j}^{(s)} C_{i-1}^{(s)}+\sum_{l=i+m-1}^{j+m-1} K_{j l}\left\{\sum_{k=-n}^{n} C_{l, l+k}^{\prime} U_{l+k}\right\}, \quad j=i+m, i+m+1, \ldots$

Изменяя далее порядок суммирования и учитывая условия $U_{i-n}=\cdots=$ $U_{i-1}=O$, получаем, что

$$
U_{j}=\sum_{s=0}^{2 m-1} P_{j}^{(s)} C_{i-1}^{(s)}+\sum_{k=i}^{i+m-1} A_{j k} U_{k}+\sum_{k=i+m}^{j+m-1+n} A_{j k} U_{k}
$$

где коэффициенты $A_{j k}$ при $j=i+m, i+m+1, \ldots$ определяются равенствами

$$
A_{j k}=\sum_{l=\alpha_{k}}^{\beta_{k}} K_{j l} C_{l k}^{\prime}, \quad k=i, i+1, \ldots, j+m-1+n,
$$

а $\alpha_{k}=\max \{k-n, i+m-1\}$ и $\beta_{k}=\min \{k+n, j+m-1\}$.

3. Справедливо следующее утверждение.

ТЕОРема 2.1. Пусть матриць $B_{j}$ удовлетворяют условиям (1.1), а матрицьл $C_{i j}(i, j=0,1, \ldots)$ таковьь, ито

$$
\sum_{j=a_{k}}^{+\infty}\left\{\sum_{l=a_{k}}^{j+m-1}\left\|K_{j l} \widetilde{C}_{l, l+k}\right\|^{2}\right\}<+\infty, \quad k=-n,-n+1, \ldots, n,
$$

әде $a_{k}=\max \{0,-k\}, \widetilde{C}_{l, l-m}=C_{l, l-m}-B_{l-m}^{*}, \widetilde{C}_{l, l+m}=C_{l, l+m}-B_{l} u \widetilde{C}_{l, l+k}=$ $C_{l, l+k}$ nри $k \neq-m$ u $k \neq m$. Тогда дефектные числа $n_{+} u n_{-}$оператора $L_{n}$ удовлетворяют условиям $n_{+}=n_{-}=m p$.

ДокАЗАтЕльство. Обозначим через $X_{1}$ пространство бесконечных последовательностей $U=\left(U_{0}, U_{1}, \ldots\right)$, где $U_{j}$ - квадратные матрицы порядка $p$, с нормой

$$
\|U\|_{1}=\left(\sum_{j=0}^{+\infty}\left\|U_{j}\right\|^{2}\right)^{1 / 2}
$$

Пусть $\Phi_{i}$ - оператор в пространстве $X_{1}$,

$$
\left(\Phi_{i} U\right)_{j}=\sum_{k=i+m}^{j+m-1+n} A_{j k} U_{k}, \quad j=i+m, i+m+1, \ldots .
$$

Стандартные вычисления показывают, что при $U, V \in X_{1}\left(U_{0}=\cdots=\right.$ $\left.U_{i+m-1}=V_{0}=\cdots=V_{i+m-1}=O\right)$ справедливы неравенства

$$
\left\|\Phi_{i} U-\Phi_{i} V\right\|_{1} \leqslant k_{i}^{\prime}\|U-V\|_{1},
$$

где

$$
k_{i}^{\prime}=\left\{\sum_{j=i+m}^{+\infty}\left[\sum_{k=i+m}^{j+m-1+n}\left\|A_{j k}\right\|^{2}\right]\right\}^{1 / 2} .
$$


Кроме того, при фиксированном $j=i+m, i+m+1, \ldots$ и $i+m \leqslant k \leqslant$ $i+m-1+n$ выполняется неравенство $\beta_{k}-\alpha_{k} \leqslant 2 n$. Следовательно, в силу известного неравенства имеем

$$
\left\|A_{j k}\right\|^{2} \leqslant(2 n+1) \sum_{l=\alpha_{k}}^{\beta_{k}}\left\|K_{j l} C_{l k}^{\prime}\right\|^{2} .
$$

Учитывая это неравенство в выражении для $k_{i}^{\prime}$, после некоторых элементарных преобразований получим, что

$$
k_{i}^{\prime} \leqslant \sqrt{2 n+1}\left\{\sum_{k=-n}^{n}\left[\sum_{j=i+m}^{+\infty}\left(\sum_{l=i+m-1}^{j+m-1}\left\|K_{j l} C_{l, l+k}^{\prime}\right\|^{2}\right)\right]\right\}^{1 / 2}
$$

где $C_{l, l+k}^{\prime}=0$, если $l+k<0$.

Из условий (1.1), (2.10) и последнего неравенства следует, что при фиксированном $z \in \mathbb{C}$ число $i$ можно взять настолько большим, чтобы $k_{i}^{\prime}<1$. Применяя далее принцип сжимающих отображений, заключаем, что система (2.9), а следовательно, и система (2.8) имеют единственное решение в пространстве $X_{1}$. Таким образом, векторное уравнение $(0.2)$ соответствующей матрицы $\mathbf{J}_{n}^{\prime}$ имеет ровно $m p$ линейно независимых решений из $l_{p}^{2}$, т. е. дефектные числа $n_{+}^{\prime}$ и $n_{-}^{\prime}$ матрицы $\mathbf{J}_{n}^{\prime}$ таковы, что $n_{+}^{\prime}=n_{-}^{\prime}=m p$. Остается заметить, что дефектные числа обобщенных якобиевых матриц $\mathbf{J}_{n}^{\prime}$ и $\mathbf{J}_{n}$ равны. Теорема 2.1 доказана.

СлЕдСТВИЕ 2.1. Пусть матриць $B_{j}$ удовлетворяют условиям (1.1), матрицьл $C_{i j}(i, j=0,1, \ldots)$ таковьл, что

$$
\sum_{l=a_{k}}^{+\infty}\left\|\left(P_{l}^{(s)}\right)^{*} \widetilde{C}_{l, l+k}\right\|^{2}<+\infty, \quad s=0,1, \ldots, 2 m-1, k=-n, \ldots, n
$$

әде $a_{k}$ и $\widetilde{C}_{l, l+k}$ определяются, как в теореме 2.1. Тогда справедливо утверждение теоремь 2.1.

ДокАЗАТЕЛЬСтво. Из равенства (2.6) и из неравенства Коши-Буняковского легко извлечь, что справедливо неравенство

$$
\left\|K_{j l} \widetilde{C}_{l, l+k}\right\|^{2} \leqslant\left(\sum_{s=0}^{2 m-1}\left\|P_{j}^{(s)}\right\|^{2}\right)\left(\sum_{s=0}^{2 m-1}\left\|\left(P_{l}^{(s)}\right)^{*} \widetilde{C}_{l, l+k}\right\|^{2}\right) .
$$

Из условий следствия 2.1 и из этого неравенства вытекает, что условия (2.10) выполнены. Применяя теорему 2.1, завершаем доказательство следствия 2.1.

Заметим, что в формулировках теоремы 2.1 и следствия 2.1 явный вид обобщенной якобиевой матрицы $\mathbf{J}_{m}^{(0)}$ не играет никакой роли. По-видимому, аналогичные утверждения справедливы каждый раз, когда известно, что для матрицы $\mathbf{J}_{m}$ имеет место вполне неопределенный случай, а матрица $\mathbf{J}_{n}$ в некотором смысле является возмущением матрицы $\mathbf{J}_{m}$.

В следующих трех следствиях теоремы 2.1 учитывается явный вид обобщенной якобиевой матрицы $\mathbf{J}_{m}^{(0)}$. 
СЛЕДСТВИЕ 2.2. Пусть матрииь $B_{j}$ удовлетворяют условиям (1.1), a матрицьл $C_{i j}(i, j=0,1, \ldots)$ таковьь, что при любом $k \in\{-n,-n+1, \ldots, n\}$ выполняются условия

$$
\sum_{i=n}^{+\infty} \sum_{j=0}^{+\infty}\left\|B_{i+2 j m}^{-1} B_{i+(2 j-1) m}^{*} \cdots B_{i+m}^{*} B_{j}^{-1} \widetilde{C}_{i, i+k}\right\|^{2}<+\infty .
$$

Тогда справедливо утверждение теоремь 2.1.

ДокАЗАТЕЛЬСтво. Изменив порядок суммирования в левых частях условий (2.10) и применив формулы (2.7), находим, что если выполнены условия (2.11), то выполняются и условия (2.10).

СлЕДСТВИЕ 2.3. Пусть матрииь $B_{j}$ удовлетворяют условиям следствия 1.2, а матрицьл $C_{i j}(i, j=0,1, \ldots)$ таковьи, что

$$
\sum_{j=n}^{+\infty}\left\|B_{j}^{-1} \widetilde{C}_{j, j+k}\right\|^{2}<+\infty, \quad k=-n,-n+1, \ldots, n .
$$

Тогда дефектные числа $n_{+} u n_{-}$оператора $L_{n}$ удовлетворяют условиям $n_{+}=n_{-}=m p$.

ДокАЗАТЕльство. Как мы уже отмечали, из условий следствия 1.2 вытекает, что условия (1.1) выполняются. Кроме того, $\left\|B_{j} B_{j+m}^{*^{-1}}\right\|<1$, и поэтому при всех $j>j_{0}$ для некоторого номера $j_{0}$ в силу формул (1.4) выполняется неравенство

$$
\left\|\left(P_{j}^{(s)}\right)^{*} \widetilde{C}_{j, j+k}\right\| \leqslant\left\|B_{j}^{-1} \widetilde{C}_{j, j+k}\right\|, \quad k=-n,-n+1, \ldots, n .
$$

Теперь в силу условий (2.12) все условия следствия 2.1 выполнены. Следствие 2.3 доказано.

СЛЕДСТВИЕ 2.4. Пусть матрищъ $B_{j}$ удовлетворяют условиям (1.6) $u$ (1.7), а матрицьь $C_{i j}(i, j=0,1, \ldots)$ таковьь, ито

$$
\sum_{i=n}^{+\infty} \frac{\left\|\widetilde{C}_{i, i+k}\right\|^{2}}{\left\|B_{i-m}\right\|}\left(\sum_{j=0}^{+\infty} \frac{1}{\left\|B_{i+(2 j+1) m}\right\|}\right)<+\infty .
$$

Тогда дефектнье числа оператора $L_{n}$ удовлетворяют условиям $n_{+}=$ $n_{-}=m p$.

ДОКАЗАТЕЛЬСТво. Согласно следствию 1.3 , матрицы $B_{j}$ удовлетворяют условиям (1.1). Кроме того, рассуждения, приведенные при доказательстве того же следствия 1.3, показывают, что из условий (1.6) следует справедливость неравенств

$$
\left\|B_{i+2 j m}^{-1} B_{i+(2 j-1) m}^{*} \cdots B_{i+m}^{*} B_{j}^{-1}\right\| \leqslant\left(\left\|B_{i-m}\right\| \cdot\left\|B_{i+(2 j+1) m}\right\|\right)^{-1 / 2} .
$$

Поэтому общий член двойного ряда в левой части условий (2.11) не превосходит величину $\left\|\widetilde{C}_{i, i+k}\right\|^{2} /\left\|B_{i-m}\right\| \cdot\left\|B_{i+(2 j+1) m}\right\|$.

Таким образом, условия (2.13) влекут за собой (2.11). Следствие 2.4 доказано. 
4. Справедлива

TEOPEMA 2.2. Пусть $\lambda \in \mathbb{C}, \operatorname{Im} \lambda>0(\operatorname{Im} \lambda<0) u$

$$
\limsup _{j \rightarrow+\infty} \sum_{k=1}^{n}\left\{\left\|\left(C_{j j}-\bar{\lambda} I\right)^{-1} C_{j, j-k}\right\|+\left\|\left(C_{j j}-\bar{\lambda} I\right)^{-1} C_{j, j+k}\right\|\right\}<1 .
$$

Тогда верхнее (нижнее) дефектное число $n_{+}\left(n_{-}\right)$оператора $L_{n}$ равно нулю.

ДокАзАтЕльство. Как и при доказательстве теоремы 2.1, пусть $X_{2}-$ пространство последовательностей матриц $U_{j}$ с нормой

$$
\|U\|_{2}=\sup _{j}\left\|U_{j}\right\|,
$$

а $G_{i}$ - оператор в пространстве $X_{2}$,

$$
\left(G_{i} U\right)_{j}=\sum_{k=1}^{n}\left\{\left(\bar{\lambda} I-C_{j j}\right)^{-1} C_{j, j-k} U_{j-k}+\left(\bar{\lambda} I-C_{j j}\right)^{-1} C_{j, j+k} U_{j+k}\right\},
$$

где $U_{i-n}=\cdots=U_{i-1}=O$. Тогда

$$
\left\|G_{i} U-G_{i} V\right\|_{2} \leqslant k_{j}^{\prime \prime}\|U-V\|_{2},
$$

где

$$
k_{i}^{\prime \prime}=\sup _{j \geqslant i} \sum_{k=1}^{n}\left\{\left\|\left(C_{j j}-\bar{\lambda} I\right)^{-1} C_{j, j-k}\right\|+\left\|\left(C_{j j}-\bar{\lambda} I\right)^{-1} C_{j, j+k}\right\|\right\} .
$$

Из условия (2.14) следует, что число $i$ можно взять настолько большим, чтобы $k_{i}^{\prime \prime}<1$. Тогда, очевидно, матричное уравнение $(2.8)$ при $z=\bar{\lambda}$ имеет в пространстве $X_{2}$ только тождественно нулевое решение. Следовательно, соответствующее векторное уравнение (0.2) имеет только тождественно нулевое решение в $l_{p}^{2}$. Теорема 2.2 доказана.

СЛЕДСТВИЕ 2.5. Пусть, начиная с некоторого $j_{0}$, все матрищь $C_{j j}$ обраmuмbl, $\lim _{j \rightarrow+\infty}\left\|C_{j j}^{-1}\right\|=0 u$

$$
\limsup _{j \rightarrow+\infty} \sum_{k=1}^{n}\left\{\left\|C_{j j}^{-1} C_{j, j-k}\right\|+\left\|C_{j j}^{-1} C_{j, j+k}\right\|\right\}<1 .
$$

Тогда оператор $L_{n}$ самосопряжен.

ДокАЗАтЕльство. В рассматриваемом случае, очевидно, существует число $\rho>0$, такое, что условие (2.14) выполняется при $\lambda \in\{\lambda:|\lambda| \leqslant \rho\}$. Поэтому, согласно теореме 2.2 , дефектные числа оператора $L_{n}$ удовлетворяют условиям $n_{+}=n_{-}=0$. Следствие 2.5 доказано.

5. Отметим, что для обыкновенных дифференциальных уравнений второго порядка известно несколько теорем, близких по содержанию к следствию 2.1 (см. [8, гл. XI, §8 и примечания к нему]). Добавим также, что основные результаты работы $[6, \mathrm{I}]$ для обобщенных якобиевых матриц следуют из теоремы 2.2 настоящей работы. 


\section{§3. Индексы дефекта некоторых обыкновенных дифференциальных операторов с полиномиальными коэффициентами}

1. Символами $H_{k}(x)(k=0,1, \ldots)$, как обычно, обозначим классические ортогональные полиномы Чебышёва-Эрмита. Пусть, далее,

$$
\varphi_{k}(x)=\left(2^{k} k ! \sqrt{\pi}\right)^{-1 / 2} H_{k}(x) e^{-x^{2} / 2}, \quad k=0,1, \ldots,
$$

- полная ортонормированная система функций Чебышёва-Эрмита в пространстве $\mathscr{L}_{2}$. Дифференциальные операции $a^{*}$ и $a$, введенные в п. $3 \S 0$ настоящей работы, действуют на функции $\varphi_{k}$ согласно формулам

$$
a^{*} \varphi_{k}=\sqrt{k+1} \varphi_{k+1}, \quad a \varphi_{k}=\sqrt{k} \varphi_{k-1}, \quad k=0,1, \ldots
$$

Эти формулы легко следуют из соотношений для полиномов $H_{k}$ и хорошо известны.

Пусть теперь $D(L)$ - область определения минимального замкнутого оператора $L$, порожденного выражением (0.4). Покажем, что

$$
\varphi_{k}(x) \in D(L), \quad k=0,1, \ldots
$$

Действительно, пусть $w(x)$ - вещественная бесконечно дифференцируемая финитная функция на всей оси, удовлетворяющая неравенствам $0 \leqslant$ $w(x) \leqslant 1$ и равная единице при $|x|<1$. Легко видеть, что $w(x / n) \varphi_{k}(x) \rightarrow$ $\varphi_{k}(x)$ и $l\left[w(x / n) \varphi_{k}(x)\right] \rightarrow l \varphi_{k}(x)$ при $n \rightarrow+\infty$ в пространстве $\mathscr{L}_{2}$. Таким образом, справедливы включения (3.2).

Из (3.2) следует, что ортонормированный базис $\varphi_{k}(x)(k=0,1, \ldots)$ пространства $\mathscr{L}_{2}$ является базисом матричного представления оператора $L$ (см. $[5$, т. 1, гл. IV, п. 53]). Используя далее представление (0.5) и соотношения (3.1), заключаем, что бесконечная эрмитова матрица, которая получается при матричном представлении дифференциальных операторов с полиномиальными коэффициентами в базисе $\varphi_{k}$, является обобщенной якобиевой матрицей.

2. Рассмотрим следующий пример:

ПримеР 3. Пусть $s$ и $m$ - целые неотрицательные числа, $h \in \mathbb{C}, h \neq 0$, и

$$
l_{0}=h a^{*^{s+m}} a^{s}+\bar{h} a^{*^{s}} a^{s+m} .
$$

Из равенств (3.1) следует, что

$l_{0} \varphi_{k}=0$, если $k<s, \quad l_{0} \varphi_{k}=\frac{h \sqrt{k !(k+m) !}}{(k-s) !} \varphi_{k+m}$, если $s \leqslant k<s+m$,

и

$$
l_{0} \varphi_{k}=\frac{\bar{h} \sqrt{(k-m) ! k !}}{(k-s-m) !} \varphi_{k-m}+\frac{h \sqrt{k !(k+m) !}}{(k-s) !} \varphi_{k+m}, \quad \text { если } k \geqslant s+m .
$$


Таким образом, если не учитывать первые $s$ тождественно нулевых строк и столбцов, то матричное представление минимального оператора $L_{0}$, порожденного выражением $l_{0}$ в базисе $\varphi_{k}(k=0,1, \ldots)$, имеет вид якобиевой матрицы $\mathbf{J}_{m}^{(0)}$ (см. п. $\left.1 \S 1\right)$ с числовыми элементами

$$
b_{k}=\frac{h \sqrt{(k+s) !(k+s+m) !}}{k !}, \quad k=0,1, \ldots
$$

Легко видеть, что $\left|b_{k}\right| \sim|h| k^{s+m / 2}$ при $k \rightarrow \infty$. Поэтому если $m \geqslant 1$ и $2 s+m>2$, то условие (1.7) следствия 1.3 выполняется. Кроме того, простые рассуждения показывают, что последовательность $\left|b_{k-m}\right| /\left|b_{k}\right| \quad(k=$ $m, m+1, \ldots)$ является неубывающей. Таким образом, условия (1.6) следствия 1.3 также выполняются. Итак, мы доказали, что при $m \geqslant 1$ и $2 s+m>2$ для матрицы $\mathbf{J}_{0}^{(0)}$ имеет место вполне неопределенный случай, в частности, дефектные числа оператора $L_{0}$ удовлетворяют условиям $n_{+}=n_{-}=m$. Эти равенства, очевидно, справедливы и в случае $m=0$, поскольку тогда только элементы главной диагонали обобщенной якобиевой матрицы $\mathbf{J}_{m}^{(0)}$ не равны нулю.

В случаях же $s=0, m=1$ или $m=2$ из признака Карлемана следует, что $n_{+}=n_{-}=0$.

3. Пусть теперь в примере $3 s=0$, а $m>2$ - четное натуральное число. Тогда коэффициент при производной $m$-го порядка в дифференциальном выражении $l_{0}$ равен $\operatorname{Re} h$. Следовательно, в случае $\operatorname{Re} h=0$ оператор $L_{0}$ является замкнутым симметрическим оператором, порождаемым дифференциальным выражением $l_{0}$ порядка $m-1$, в пространстве $\mathscr{L}_{2}$ с дефектными числами $n_{+}=n_{-}=m$. Ясно, что аналогичные примеры можно построить и в случае нечетного $m>2$. Таким образом, дефектные числа оператора $L_{0}$ могут оказаться и больше, чем порядок выражения $l_{0}$. Причины этого явления очевидны: коэффициент при наивысшей производной в выражении $l_{0}$ обращается в нуль при $x=0$. Kак мы уже отмечали в п. $2 \S 0$, указанное явление в случае обобщенных якобиевых матриц не имеет места.

Вернемся к случаю, когда $m \geqslant 1$ и $2 s+m>2$. Легко установить, что матричным представлением для минимального оператора, порожденного выражением $l_{0}^{k}(k \in\{1,2, \ldots\})$, служит обобщенная якобиева матрица $\left(\mathbf{J}_{m}^{(0)}\right)^{k}$. Можно доказать, что и для этой матрицы имеет место вполне неопределенный случай. С другой стороны, как мы уже отмечали во введении, операторы, отвечающие вполне неопределенным якобиевым матрицам, являются целыми операторами минимального типа (см. $[1,2])$ и обладают рядом замечательных свойств. Таким образом, оператор, порожденный дифференциальным выражением $l_{0}^{k}$, является целым оператором минимального типа с дефектными числами $n_{+}=n_{-}=k m$, а порядок этого выражения может отличаться от $k(s+2 m)$ и зависит еще от $h$. Приведем примеры.

Пример 4. (а) Пусть $s=0, m=3, h \neq 0$ и $h=\sqrt{2}(\alpha-2 \beta i)$. Тогда

$$
l_{0} f=\alpha\left[3\left(x f^{\prime}\right)^{\prime}+x^{3} f\right]+i \beta\left\{2 f^{\prime \prime \prime}+3\left[\left(x^{2} f\right)^{\prime}+x^{2} f^{\prime}\right]\right\} .
$$


(б) Пусть $s=1, m=1, h \neq 0$ и $h=-\sqrt{2}(\alpha-2 \beta i)$. Тогда

$$
l_{0} f=\alpha\left[\left(x f^{\prime}\right)^{\prime}-\left(x^{3}-2 x\right) f\right]+i \beta\left\{2 f^{\prime \prime \prime}-\left[\left(\left(x^{2}-2\right) f\right)^{\prime}+\left(x^{2}-2\right) f^{\prime}\right]\right\} .
$$

Порядок дифференциального выражения $l_{0}$ равен двум, если $\beta=0$, и трем во всех остальных случаях. При этом дефектные числа оператора, порожденного выражением $l_{0}^{k}$, удовлетворяют условиям $n_{+}=n_{-}=3 k$ в случае (a) и $n_{+}=n_{-}=k$ в случае (б).

4. Отметим, что результаты $\S 2$ позволяют определить индексы дефекта некоторых дифференциальных операторов, порожденных выражениями вида $l_{0}+l_{1}$, где $l_{0}$ определено выше, а $l_{1}$ имеет вид

$$
l_{1}=\sum_{i+j<2 s+m} h_{i j} a^{*^{i}} a^{j}, \quad h_{i j}=\bar{h}_{j i} .
$$

Однако якобиевы матрицы, возникающие при матричном представлении дифференциальных операторов с полиномиальными коэффициентами, имеют ряд специфических свойств. Эти свойства позволяют усилить некоторые результаты $§ 2$ настоящей работы для соответствующих матриц. Таким путем, по-видимому, можно получить более исчерпывающую информацию о дефектных числах и других спектральных свойствах дифференциальных операторов, и мы планируем посвятить этому кругу вопросов отдельную работу.

\section{ЛИТЕРАТУРА}

1. Крейн М. Г. Основные положения теории представления эрмитовых операторов с индексом дефекта $(m, m)$. Укр. матем. ж., № 2, 3-66 (1949).

2. Крейн М. Г. Бесконечные Ј-матрицы и матричная проблема моментов. ДАН СССР, т. 69, № 3, 125-128 (1949).

3. Березанский Ю. М. Разложение по собственным функциям самосопряженных операторов. Наукова думка, Киев, 1965.

4. Костюченко A. Г., Мирзоев K. A. Трехчленные рекуррентные соотношения с матричными коэффициентами. Вполне неопределенный случай. Матем. заметки, 63, вып. 5, 709-716 (1998).

5. Ахиезер Н. И., Глазман И. М. Теория линейных операторов в гильбертовом пространстве. Т. 1, 2, Харьков, 1977, 1978.

6. Чистяков A. Л. Индексы дефектов симметрических операторов в прямой сумме гильбертовых пространств. I. Вестник МГУ, сер. матем. мех., № 3, 5-21 (1969); II, там же, № 4, 3-5 (1969).

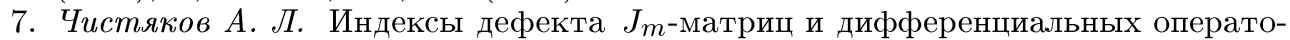
ров с полиномиальными коэффициентами. Матем. сб., 85 (127), № 4(8), 474-503 (1971).

8. Хартман Ф. Обыкновенные дифференциальные уравнения. Мир, М., 1970.

Московский государственный университет им. М. В. Ломоносова, механико-математический факультет

Московский государственный авиационный технологический университет им. К. Э. Циолковского 